\title{
Improved phenomenological equation of state in the chemical picture
}

\author{
Regner Trampedach
}

\author{
Mt. Stromlo Observatory, Australian National University
}

\begin{abstract}
I present an overview of an equation of state, being developed in the chemical picture, and based on the very successful MHD equation of state. The flexibility of the chemical picture combined with the free-energy minimization procedure, makes it rather straight-forward, albeit laborious, to include new effects in the model free-energy, simply by adding new terms.

The most notable additions to the original MHD equation of state, are relativistic effects, quantum effects, improved higher order Coulomb terms and a long list of molecules other than the $\mathrm{H}_{2}$ and $\mathrm{H}_{2}^{+}$treated so far.
\end{abstract}

\section{INTRODUCTION}

Our understanding of stellar structure and evolution is the foundation for most astrophysical endeavors, whether related to clusters of stars, galaxies, the early universe or the distance scale. The quality of stellar modeling therefore has an impact beyond its own field. Our stellar models, in turn, depend crucially on the physics they are based on, of which there are two main categories; dynamical processes, e.g., convection and rotation, and micro-physics, e.g., the equation of state, opacities and nuclear reaction rates.

The late 80'es and early 90'es saw great advances in equation of state and opacity calculations for astrophysical plasmas and the published tables [1, 2, 3, 4] were immediately put to use. The improved atomic physics resolved some longstanding problems, e.g., the disagreement between the theoretical evolutionary and observed dynamical masses of Cepheids and RR Lyraes [5, 6, 7].

At the same time, the increase in computer-power also enabled the emergence of realistic, 3-dimensional simulations of convection [8], convection being the most important of the dynamical processes. Due to the time-scales involved these simulations are constrained to the outer layers of stars. In the deeper layers, however, the stratification of the convection zone is adequately described by the mixing-length formulation [9]. The main parameter, $\alpha$, determining the asymptotic adiabat of the convection zone, is not prescribed by this formulation. The 3D simulations can be used to calibrate $\alpha$, and to describe the stratification in the upper layers where convective fluctuations are in the non-linear regime, and where radiation escapes the stellar surface.

The transition from diffusive to free-streaming radiative transfer (i.e., the photosphere) is dealt with in great detail in conventional 1D stellar atmosphere calculations, where nowadays $10^{5}-10^{6}$ wavelengths are used and non-LTE effects are included when necessary. Convection, however, is described in the mixing-length formulation which 
fails in the surface layers. The only alternative that can result in realistic and selfconsistent stratifications, spectral line-shapes[10], abundances [11], etc., are the convection simulations. It has recently been shown that a simplified radiative transfer scheme for the simulations, can reproduce the monochromatic solution, but for a small fraction of the computational cost [12]. Apart from non-LTE effects, the radiative transfer in the simulations can rival that of modern 1D atmosphere calculations, but with a realistic and consistent treatment of convection.

The convection simulations are deeper than normal atmosphere models, since, in general, convection turns adiabatic in deeper layers than where radiative transfer turns diffusive. As a consequence, the convection simulations straddle the regimes of a simple Saha equation of state that includes molecules, and a more sophisticated one that, however, can neglect molecules (other than $\mathrm{H}_{2}$ ). The desire to accurately match the convection simulations with interior models, further promotes the concept of a stellar equation of state that includes everything from molecules at low temperature, interaction effects at high densities and relativistic effects at high temperatures. The present paper is an outline of such work in progress.

\section{THE MHD EQUATION OF STATE}

The MHD equation of state [13, 14] was conceived as part of the International Opacity Project (OP) [15, 16], of the late 1980's, to calculate precise and accurate opacities for stellar envelopes. The emphasis was on detailed quantum-mechanical calculations of the electron structure of all ions of the astrophysically most important elements. The resulting energy-levels and effective radii were used in the MHD equation of state and the transition probabilities were used for calculation of absorption coefficients for each state of each atom/ion. Combining the population of these states, as found from the equation of state, with the absorption coefficients, then results in the opacity; a selfconsistent set of calculations.

The OPAL project [3, 4] is a parallel and similar equation of state and opacity effort, but independent of MHD and OP, and pursued in the physical picture.

The key concept introduced in the MHD equation of state, was that of occupation probabilities, $w_{i}$, of a particular state, $i$ [17]. The Boltzmann-factors in conventional calculations of the population of states, are based on the assumption of an isolated particle and therefore ignore the presence of neighboring particles. This results in the well-known $24 \%$ un-ionized hydrogen at the center of the Sun, in this simplistic picture. It is obvious that the density at the Solar center leaves no room for hydrogen atoms. Two methods were introduced to cut-off the summation of the otherwise divergent partition function; one considered the available volume and the size of bound states and assumed dense packing, the other used a lowering of the ionization potential to spill bound states into the continuum. Both methods introduce discontinuities into the equation of state and most implementations have lacked internal consistency.

The MHD equation of state, on the other hand, includes a realistic model of a physical process that directly leads to ionization; ionization by repeated crossings of Starkmanifolds by fluctuating electric fields, as measured by [18]. This leads to ionization 
when the amplitude of the fluctuating field exceeds a threshold, $F_{\mathrm{cr}}$, for given state. The probability of state $i$ being present in a plasma is therefore

$$
w_{i}=Q\left(F_{\mathrm{cr}, i}\right)=\int_{0}^{F_{\mathrm{cr}, i}} P(F) \mathrm{d} F
$$

where $P(F)$ is the micro-field distribution. This provides us with a smooth, selfconsistent and physically plausible equation of state.

A further advantage over previous work is the analytical derivatives, which ensures smooth and consistent thermodynamic derivatives.

\section{BEYOND THE ORIGINAL MHD}

The improvements detailed below, are compared to the original MHD and to the OPAL equation of state[3] in Figs. 1, 3] These plots show pressure differences in the left-hand panel and $\gamma_{1}$ differences in the right-hand panel. These quantities have been selected for their importance for the hydrostatic structure and the sound-speed profile, respectively. In these plots the solid line shows the difference (new MHD-original MHD) as the solid lines, (OPAL-original MHD) as the gray lines. The dotted lines show the original MHD (as published) with the $\tau$-function that was introduced to curb the otherwise diverging (with density) Debye-Hückel term. This $\tau$-factor was discussed by [19], disputing its physical foundation and showing that better agreement with OPAL is obtained using $\tau=1$, as shown with the gray lines in Figs. 1 3 The solid, the dotted and the gray lines are the same in all three plots. The dashed lines show the effect of turning off some of the improvements, one at a time.

The OPAL equation of state is an important comparison case, since Solar models based on it show overall better agreement with helioseismic inversions [20], compared to MHD. Looking more carefully at the outer layers of the Sun, using high degree pmodes, models using the MHD equation of state seems to be closer to the Sun in the outer 7\%, than ones using OPAL [21]. This region includes the $\mathrm{H}-, \mathrm{He}-$ and some of the $\mathrm{He}^{+}$-ionization zone, and covers the left half of each of the plots in Figs. 1-3 Deeper in the Sun we therefore want better agreement with OPAL (i.e., the solid curve close to the gray curve in Figs. 13), whereas the situation is less clear further out.

\section{Micro-field Distributions}

The original MHD formulation used linear fits to the Holtzmark distribution, which assumes non-interacting particles. [22] introduced much improved fits to a micro-field distribution model that accounts for particle interactions through the Debye-Hückel theory. I have included this so-called $Q$-MHD formulation and the effect under Solar circumstances can be seen in Fig. 1 The effect is largest in the derivatives, where a lot of new structure is introduced. This change will most likely have a larger effect on the opacity since it changes the population of highly excited states that are often strong absorbers. 

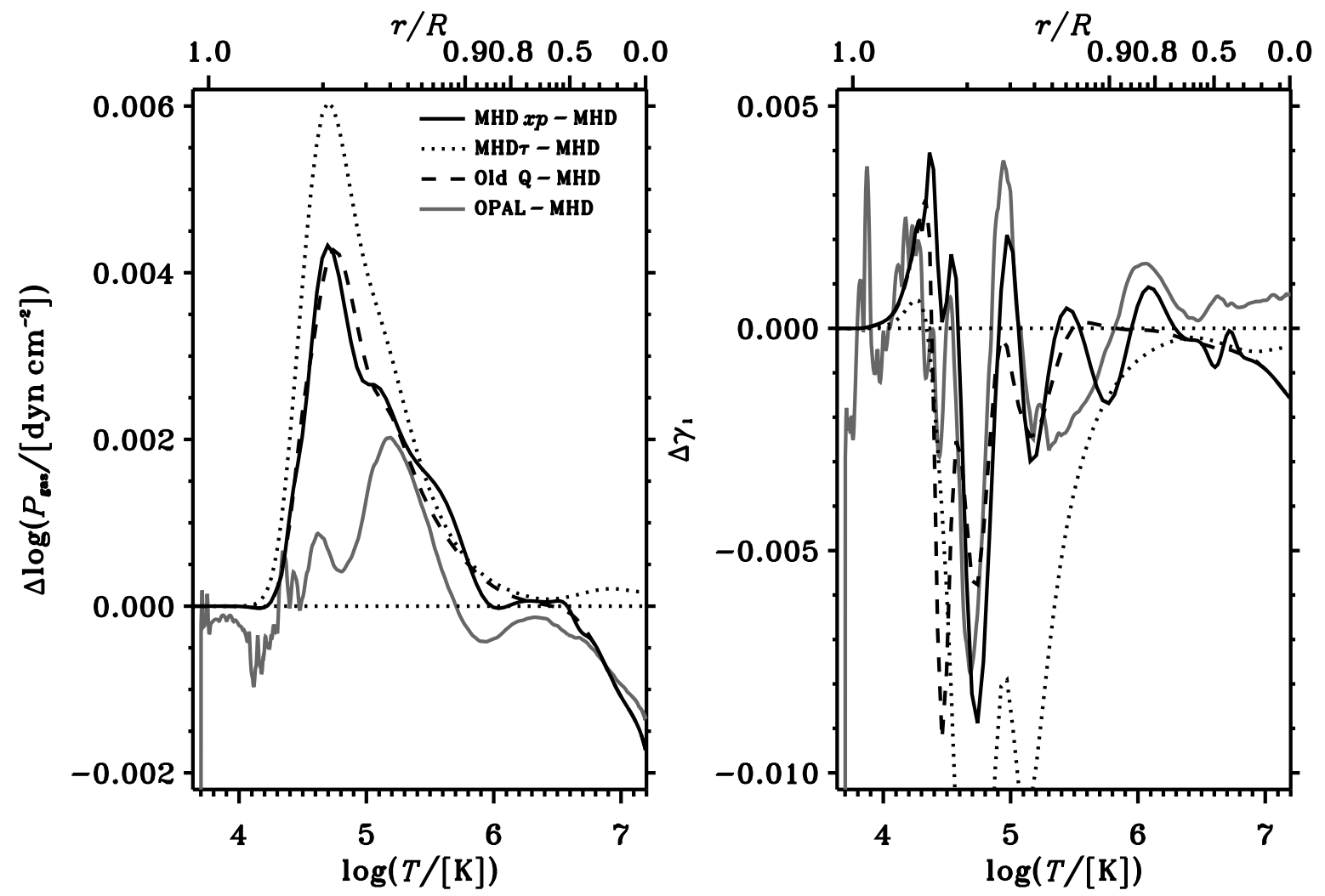

FIGURE 1. Differences between original MHD and: OPAL (gray), all changes (solid), all changes except old micro-field distribution (dashed), along a Solar $\rho / T$-stratification.

\section{Quantum effects}

Apart from the effect on particle statistics, in the form of degeneracy, there are at least two other effects of quantum mechanics that needs to be included; quantum diffraction and exchange interactions. The former is a consequence of Heisenberg's uncertainty relation and the realization that particles are wave-packets of finite size. This results in finite charge-densities and therefore avoids the short-range divergence of the Coulomb potential. This effect replaces the $\tau$-factor mentioned above.

The exchange term arise from Pauli's exclusion principle between identical particles; the wave-functions of two identical particles will either overlap or repel each other, depending on their spin, thereby changing their interaction energy compared to that of differing particles. The effect of the first-order exchange-term [23] is shown in Fig. [2]

\section{Interactions with Neutral Particles}

In the original MHD equation of state neutral particles were treated as hard spheres, but only to first order in particle density, $N_{i}$. The second order term was included 

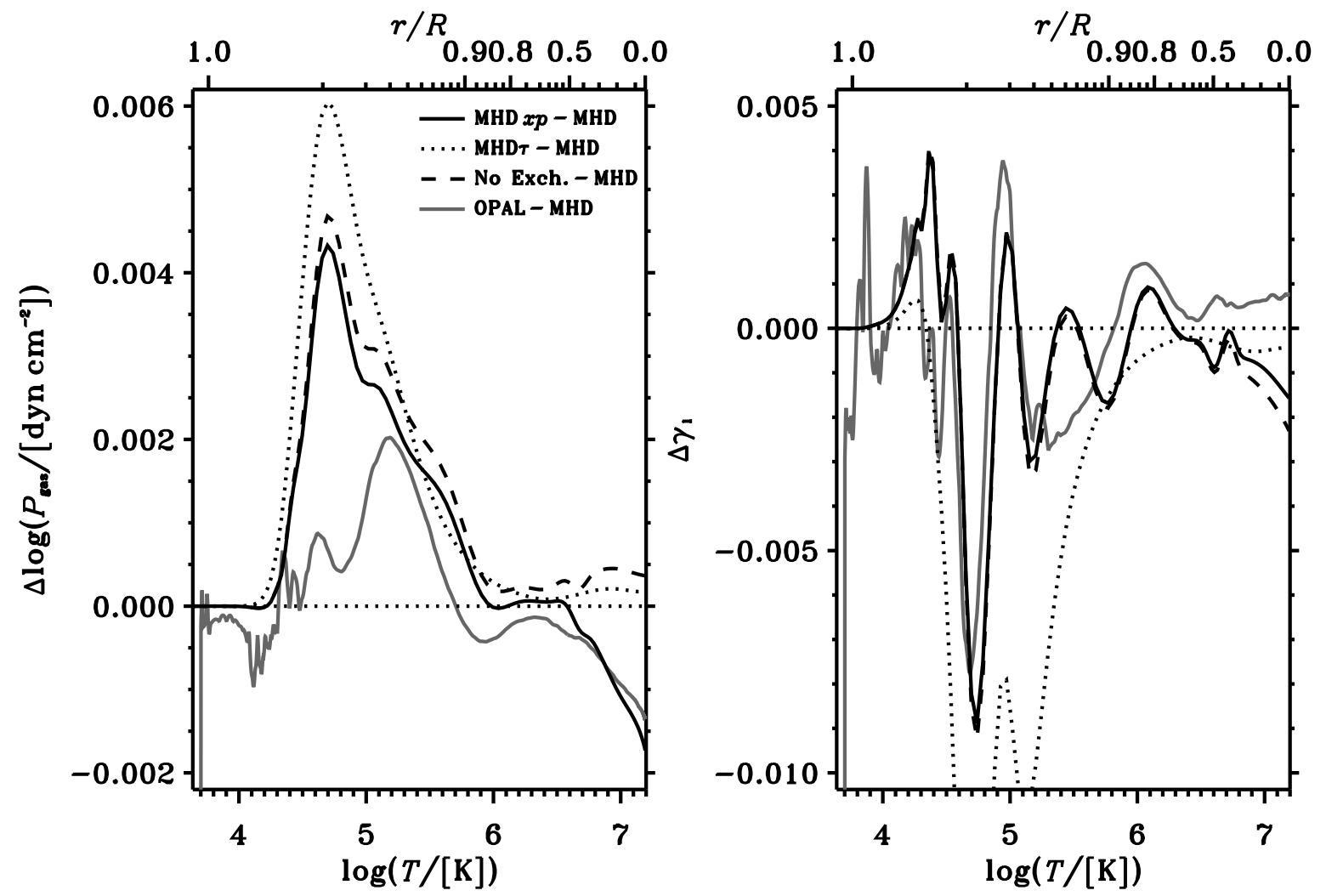

FIGURE 2. Differences between original MHD and: OPAL (gray), all changes (solid), all changes except for exchange effects (dashed), along a Solar $\rho / T$-stratification.

in an approximate way through the $\Psi$-function discussed by [24]. Apart from these approximations, the hard-sphere model has its problems. First of all, it is undefined for high densities and can disrupt the convergence of the free-energy minimization. Second, it is un-physical and ignores the underlying forces. A neutral atom has an extended electron distribution, and in close encounters, another particle will dip into this electron wave-function and begin to feel the charge of the nucleus which is no longer completely screened. At high densities net-neutral particles will therefore have a small effective charge. The nice thing about this model, is that it also applies to partially stripped ions, and all particles are treated on an equal footing. Under Solar circumstances this change has no discernible effect, but it most likely will for cooler stars.

\section{Coulomb Interactions}

By far the largest change-at least in the Sun-arise from including higher-order terms in the Coulomb interactions, beyond the Debye-Hückel term, $F_{\mathrm{DH}}$. The original MHD had a $\tau$-correction to $F_{\mathrm{DH}}$ that turned out to be un-physical [19], and is now replaced by a correction-factor based on Monte-Carlo simulations of the one-component- 

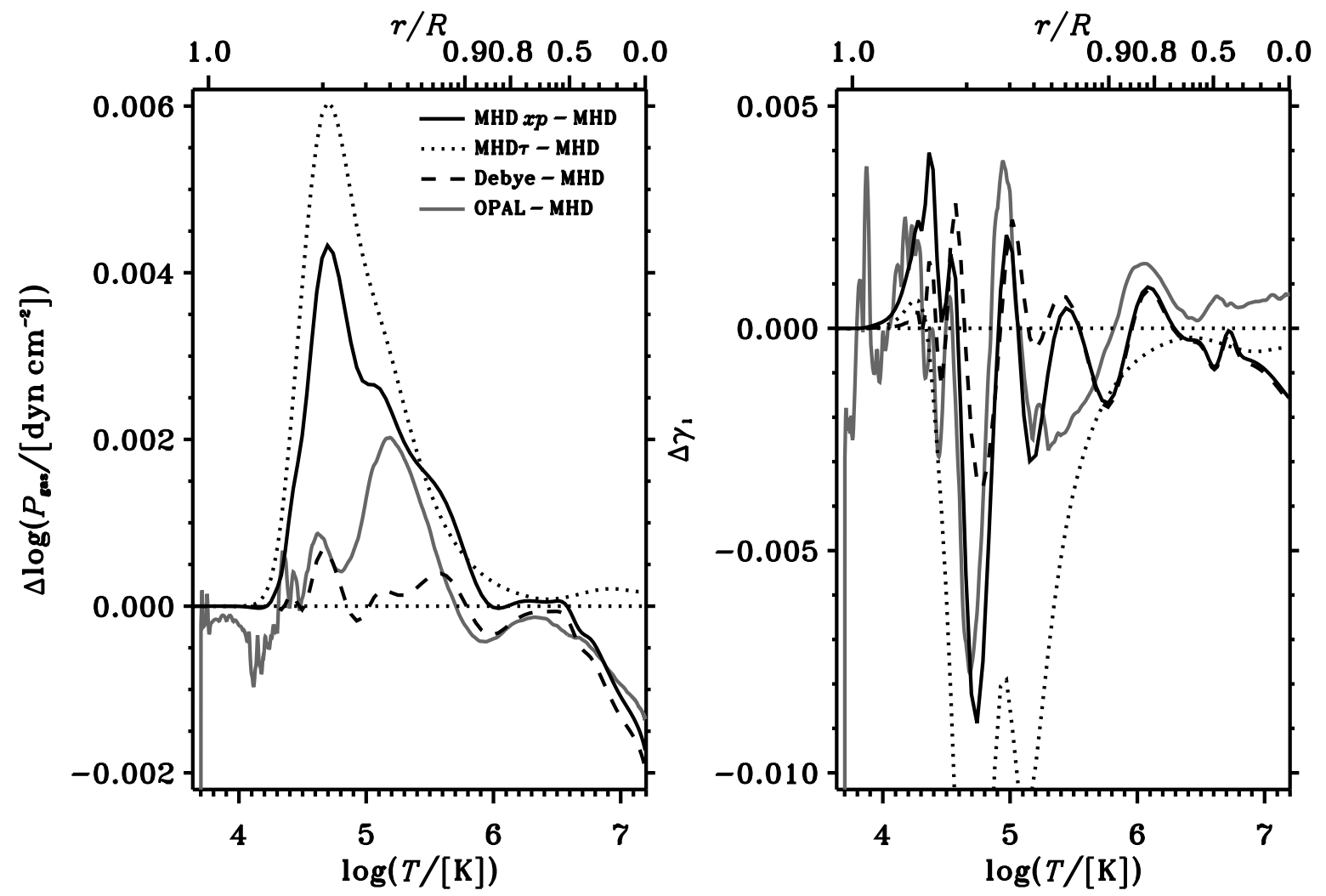

FIGURE 3. Differences between original MHD and: OPAL (gray), all changes (solid), all changes except pure Debye-Hückel (dashed), along a Solar $\rho / T$-stratification.

plasma [25], implicitly including many-body interactions. The effect of this change can be seen in Fig. 3, it is constrained to the convection zone and peaks at a depth of about $7 \mathrm{Mm}$, which is surprisingly close to the surface.

\section{Additional Changes}

Relativistically degenerate electrons are included as detailed by [26]. The effect is very small except in the adiabatic exponent, $\gamma_{1}$, which is lowered appreciably in the inner half of the Sun.Relativistic effects are not included in the version of OPAL we have used for comparison, but omitting it in the new MHD results in very good agreement with OPAL for $\log T>6.7$. The new version of OPAL includes relativistic electrons, as well as a few other improvements [27].

Molecules are included by means of the fits to partition functions, compiled by [28], augmented by a parametrized pressure dissociation, based on the detailed treatment by MHD of the $\mathrm{H}_{2}$ - and $\mathrm{H}_{2}^{+}$-molecules. Molecules are selected from a list of 315 di-atomic and 99 poly-atomic molecules, depending on the elements included in the equation of state calculation. This change will mostly affect the atmospheric opacities. 


\section{SUMMARY}

A new equation of state is being developed, based on the MHD equation of state, but with a number of improvements that will hopefully bring it on par with the OPAL equation of state. Tables have not been computed yet, so calculations of Solar models with the new equation of state are not yet possible. Comparisons on a fixed $\rho / T$-track of a Solar model, as presented here, are encouraging, however.

\section{ACKNOWLEDGMENTS}

I am grateful to the organizers of the workshop, for the invitation to present and for a fruitful two weeks. I would also like to thank Werner Däppen for full access to the original MHD-code, including rights to and help with modifying it.

\section{REFERENCES}

1. Mihalas, D., Hummer, D. G., Mihalas, B. W., and Däppen, W., ApJ, 350, 300-308 (1990).

2. Seaton, M. J., Yan, Y., Mihalas, D., and Pradhan, A. K., M.N.R.A.S., 266, 805-828 (1994).

3. Rogers, F. J., Swenson, F. J., and Iglesias, C. A., ApJ, 456, 902-908 (1996).

4. Rogers, F. J., and Iglesias, C. A., ApJS, 401, 361-366 (1992).

5. Andreasen, G. K., $A \& A$, 201, 72-79 (1988).

6. Andreasen, G. K., and Petersen, J. O., A\&A, 192, L4-L6 (1988.).

7. Yi, S., Lee, Y.-W., and Demarque, P., ApJ, 411, L25-L28 (1993).

8. Nordlund, A., and Stein, R. F., Comput. Phys. Commun., 59, 119 (1990).

9. Böhm-Vitense, E., Zs. f. Astroph., 46, 108-143 (1958).

10. Asplund, M., Nordlund, Å., Trampedach, R., Allende Prieto, C., and Stein, R. F., A\&A, 359, 729-742 (2000).

11. Asplund, M., Nordlund, A., Trampedach, R., and Stein, R. F., A\&A, 359, 743-754 (2000).

12. Trampedach, R., and Asplund, M., "Radiative Transfer with Very Few Wavelengths," in ASP Conf. Ser. 293: 3D Stellar Evolution, 2003, pp. 209-+.

13. Mihalas, D., Däppen, W., and Hummer, D. G., ApJ, 331, 815-825 (1988).

14. Däppen, W., Mihalas, D., Hummer, D. G., and Mihalas, B. W., ApJ, 332, 261-270 (1988).

15. Seaton, M. J., editor, The Opacity Project, vol. 1, Institute of Physics Publishing, 1995.

16. Berrington, K. A., editor, The Opacity Project, vol. 2, Institute of Physics Publishing, 1997.

17. Hummer, D. G., and Mihalas, D., ApJ, 331, 794-814 (1988).

18. Pillet, P., van Linden van den Heuvell, H. B., Smith, W. W., Kachru, R., Tran, N. H., and Gallagher, T. F., Phys. Rev. A, 30, 280-294 (1984).

19. Trampedach, R., Däppen, W., and Baturin, V. A., ApJ (2004), (submitted).

20. Christensen-Dalsgaard, J., and Däppen, W., $A \& A R, 4,267-361$ (1992).

21. Di Mauro, M. P., Christensen-Dalsgaard, J., Rabello-Soares, M. C., and Basu, S., A\&A, 384, 666-677 (2002).

22. Nayfonov, A., Däppen, W., Hummer, D. G., and Mihalas, D., ApJ, 526, 451-464 (1999).

23. Kovetz, A., Lamb, D. Q., and Horn, H. M. V., ApJ, 174, 109-120 (1972).

24. Trampedach, R., and Däppen, W., ApJ (2004), (in preparation).

25. Slattery, W. L., Doolen, G. D., DeWitt, H. E., and Slattery, W. L., Phys. Rev. A, 26, 2255+ (1982).

26. Gong, Z., Däppen, W., and Zejda, L., ApJ, 546, 1178-1182 (2001).

27. Rogers, F. J., and Nayfonov, A., ApJ, 576, 1064-1074 (2002).

28. Irwin, A. W., ApJS, 45, 621+ (1981). 